\title{
Length of main left coronary artery in relation to atherosclerosis of its branches A coronary arteriographic study
}

\author{
N. Gazetopoulos, P. J. Ioannidis, A. Marselos, D. Kelekis, C. Lolas, D. Avgoustakis, and \\ C. Tountas \\ From the Cardiology Division, 2nd Department of Surgery, Aretaeion Hospital, and the Department of \\ Cardiology, Hippocrateion Hospital, Athens National University, Athens, Greece
}

The relation between the length of the main left coronary artery and the presence of atherosclerosis in its branches or the presence of complete left bundle-branch block was studied by selective coronary arteriography in 43 persons.

The length of the main left coronary artery was found to be significantly shorter in patients with coronary atherosclerosis than in subjects without angiographic evidence of coronary artery disease. In patients with electrocardiographic evidence of complete left bundle-branch block, the length of the left main coronary artery was significantly shorter than that in both previous groups.

In view of these findings, it is suggested that a short main left coronary artery should be considered as a congenital factor predisposing to the development of coronary artery disease. The possible mechanisms leading to atherosclerosis of the left coronary arterial branches in the presence of a short main trunk are discussed.

In recent studies coronary arteriographic findings were related to intraventricular conduction disturbances (Haft, Herman, and Gorling, 1969; Beach et al., 1969; Lewis et al., 1970; Hamby, Tabrah, and Gupta, 1973). A remarkable observation was made by Lewis et al. (1970), who noted that a short main left coronary artery was present in a great proportion of patients with complete left bundle-branch block. It was suggested that in the presence of a short main left coronary artery trunk, the initial part of the left anterior descending artery is exposed to unusual stress from systolic kinking, with resulting impairment of the blood supply to the bundle of His. Segments of arteries subjected to systolic kinking are known to be particularly liable to intimal changes and atherosclerosis (Fulton, 1965; Glagov, 1972).

From these observations, it might be supposed that the length of the main left coronary artery is one of the factors that may contribute to the development of atherosclerosis in its branches. The purpose of this investigation was to find out if there is any difference in the length of this artery in patients with and without angiographic evidence Receired 22 June 1975. of coronary artery disease. Some further data are also presented concerning its length in patients with complete left bundle-branch block.

\section{Subjects and Methods}

The study was based on the coronary arteriographic findings in 43 subjects. The only criterion for inclusion was the satisfactory visualization of the main left coronary artery and its branches.

The patients studied were divided into three groups. The first group included 19 patients without arteriographic evidence of coronary atherosclerosis. Twelve of these had a form of heart disease, usually aortic and mitral valve lesions, leading to left ventricular hypertrophy. The others presented with atypical findings suggestive of coronary artery disease.

The second group included 20 patients with atherosclerotic lesions in the left anterior descending or both branches of the left coronary artery. Two also had left ventricular hypertrophy caused by valvular disease. Stenotic lesions in the left anterior descending artery varied from 50 per cent to complete occlusion in 17 cases; in 3, the obstruction was of a lesser degree. In 13 atherosclerosis was also present in the circumflex branch of the left coronary artery. 
The third group contained 4 patients with complete left bundle-branch block. Three of these had atherosclerotic changes in both left coronary artery branches. The fourth had congestive cardiomyopathy without evidence of coronary artery disease.

Selective coronary arteriograms were obtained using cine and/or cut films by Judkins' or Sones' technique. Obstructive lesions were evaluated according to the percentage narrowing of the lumen. The left coronary artery was usually visualized in both right and left anterior oblique projections on a rotating table.

Measurements of the length of the main left coronary artery were made, in the diastolic phase, by at least two observers, one of whom did not know the patient. Usually the estimates of the two observers coincided and when there was disagreement the estimates differed by 1 to $2 \mathrm{~mm}$ at most. When the two measurements differed, these were averaged. Errors caused by magnification were corrected by comparing the observed with the known diameter of the catheter used. The length of the main left coronary artery was determined from its origin to the point of its bifurcation into the left anterior descending and circumflex branches in the right anterior oblique projection, as was done by previous investigators (Lewis et al., 1970; Fox, Davies, and Webb-Peploe, 1973; Kronzon, Deutsch, and Glassman, 1974). In cases where two projections perpendicular to one another were available, the spatial length was estimated by using Pythagoras' theorem. Since the course of the main left coronary artery is almost horizontal, the theorem could be applied as follows:

$$
\mathbf{V} \simeq \sqrt{\mathbf{R}^{2}+\mathbf{L}^{2}}
$$

where $\mathrm{V}=$ spatial length, and $R$ and $\mathrm{L}=$ length in right and left anterior oblique projections. When the main left coronary artery departs from the horizontal plane, its length is slightly overestimated.

\section{Statistical analysis}

Data presented a normal distribution curve with minimal positive skew. Differences between group mean values were tested for their level of significance by using the Student's t test.

\section{Results}

The Table shows mean values \pm 1 standard deviation, and ranges, of the main left coronary artery in each of the three groups, and the statistical significance of differences between the mean values.

In the group of cases without any arteriographic evidence of coronary atherosclerosis the mean of the main left coronary artery was the longest both in the RAO projection $(16.8 \pm 4.13 \mathrm{~mm})$ and in space $(19.93 \pm 3.58 \mathrm{~mm})$.

TABLE Length of main left coronary artery in three groups of patients

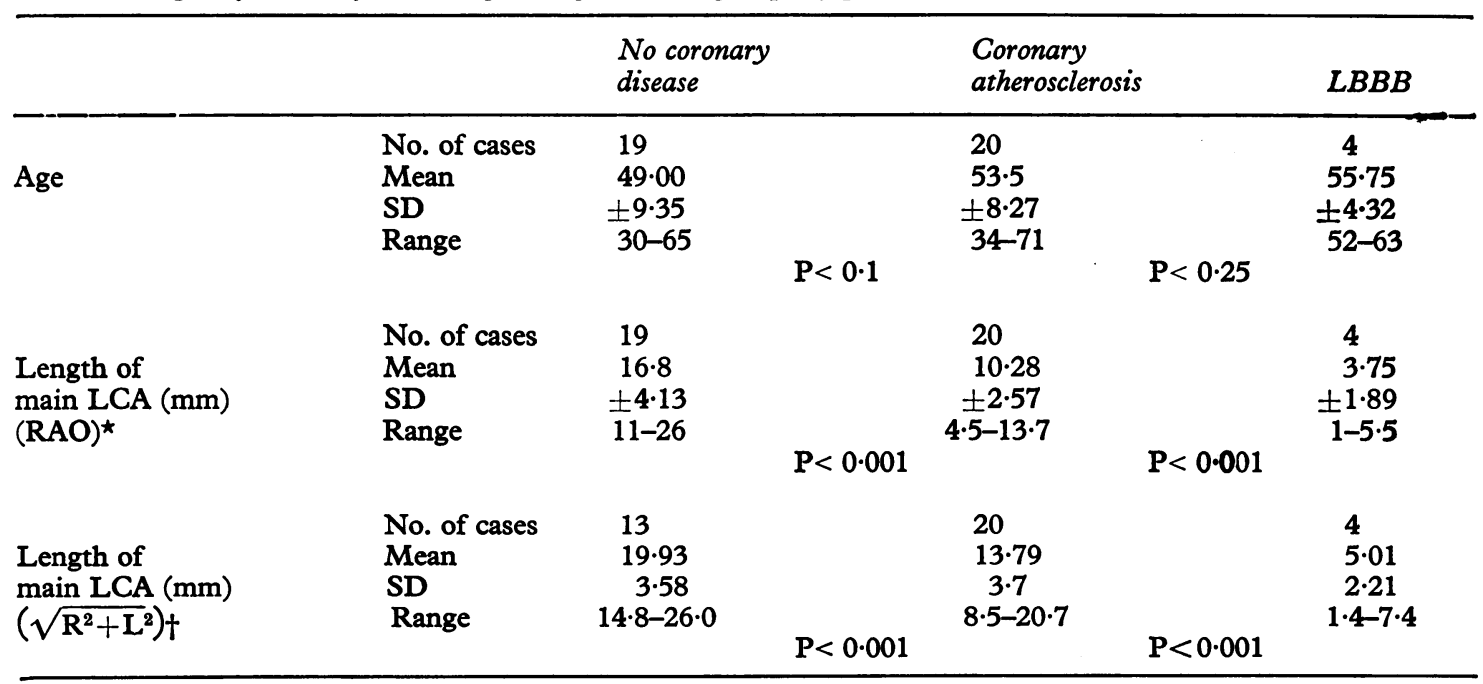

LBBB, left bundle-branch block.

*Length of main left coronary artery measured in right anterior oblique projection.

†Length of main left coronary artery in space, calculated from measurements in both oblique projections.

$P$, Probability of no significant difference between mean values. 
In the group of cases with atherosclerosis of the left coronary artery branches the mean length of the main left coronary artery was significantly shorter than in the previous group both in the RAO projection $(10.28 \pm 2.57 \mathrm{~mm}, \mathrm{P}<0.001)$ and in space $(13.79 \pm 3.7 \mathrm{~mm}, P<0.001)$.

Finally, in the 4 cases with complete left bundlebranch block the mean length of the main left coronary artery was significantly shorter than in the previous two groups, both in the RAO projection $(3.75 \pm 1.89 \mathrm{~mm}, P<0.001)$ and in space $(5.01 \pm 2.11 \mathrm{~mm}, P<0.001)$. Differences in age between the groups were not significant at the 5 per cent level.

The length of the main left coronary artery in individual patients in each group is shown in Fig. 1. Cases with left ventricular hypertrophy are represented by open symbols; the length of the main left coronary artery in these cases does not differ from that in cases without left ventricular hypertrophy. Differences between mean group values remain significant $(P<0.0025)$ after exclusion of cases with left ventricular hypertrophy.

Figs. 2 and 3 show the coronary arteriograms of two representative cases.

\section{Discussion}

The mean length of the main left coronary artery, estimated by coronary arteriography in the right anterior oblique position, as reported by different authors, varies. Lewis et al. (1970) reported a length of $12.8 \mathrm{~mm}$, Fox et al. (1973) found it to be 9.5 $\mathrm{mm}$, and Kronzon et al. (1974) $10.4 \mathrm{~mm}$. In our study the mean length of the main left coronary artery, measured in this way, was $12.4 \mathrm{~mm}$. The measurement of a three-dimensional structure in a single plane, unless parallel to it, will be an underestimate. In order to obtain a closer approximation to actual length, we also estimated the spatial length by using both the right and left anterior oblique projections. The mean length of the main left coronary artery estimated thus was found to be $15.0 \mathrm{~mm}$. In necropsy studies the mean main left coronary arterial length was found by Baroldi and Scomazzoni (1967) to be $13.5 \mathrm{~mm}$. Regardless of the reasons for the discrepancy between the reported mean values in different studies, in any one study the differences between groups are likely to be valid.

A relation between a short main left coronary

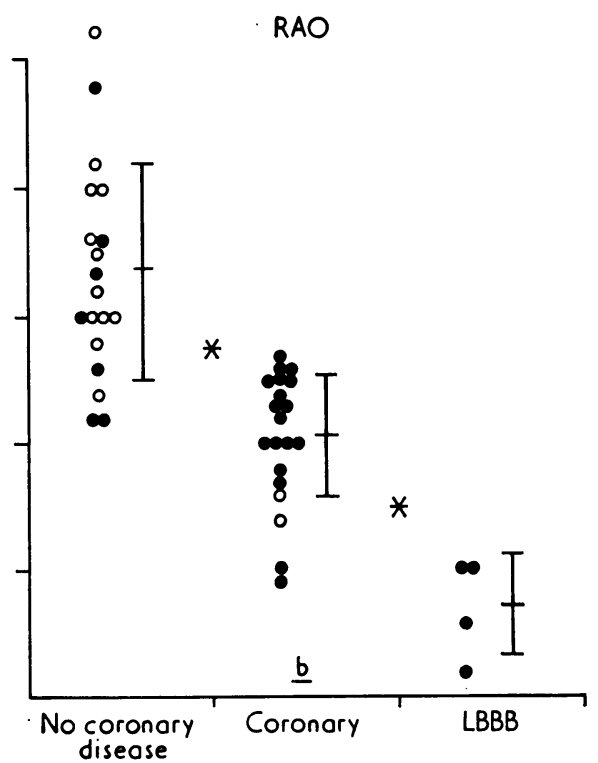

FIG. 1 Distribution of cases according to the length of the main left coronary artery, in cases without evidence of coronary atherosclerosis, cases with coronary atherosclerosis and cases with complete left bundle-branch block ( $L B B B$ ).

(a) spatial length. (b) length in the right anterior oblique projection, $\star P<0.001$ between mean values of adjacent groups. Open circles represent cases with left ventricular hypertrophy. 


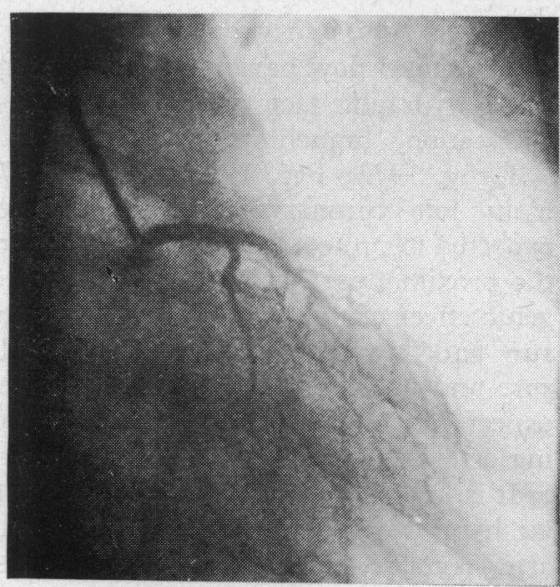

RAO

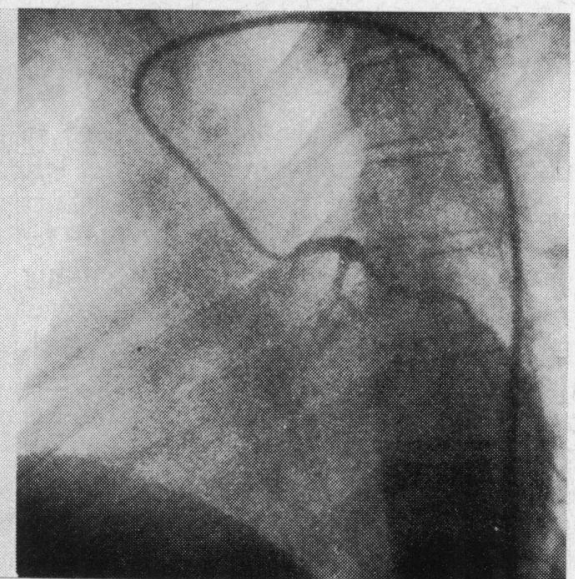

LAO

FIG. 2 Coronary arteriogram of a case without evidence of coronary atherosclerosis. Note the long course of the main left coronary artery. RAO : right anterior oblique projection. LAO: left anterior oblique projection.

artery and complete left bundle-branch block was first observed by Lewis et al. (1970) in 11 out of 12 cases. In these cases the length of the main left coronary artery trunk ranged from 0 to $6 \mathrm{~mm}$ (mean $4.5 \mathrm{~mm}$ ) in the right anterior oblique projection. In another study a main left coronary artery shorter than $6 \mathrm{~mm}$ was found in only 2 out of 8 cases with complete left bundle-branch block (Hamby et al.,
1973). No information was provided about the length of the main left coronary artery in other patients studied. All 4 patients with complete left bundle-branch block in our study had a main left coronary artery of less than $6 \mathrm{~mm}$ in length. In spite of the limited number of our cases, it seems that a complete left bundle-branch block is a common finding among individuals with a short

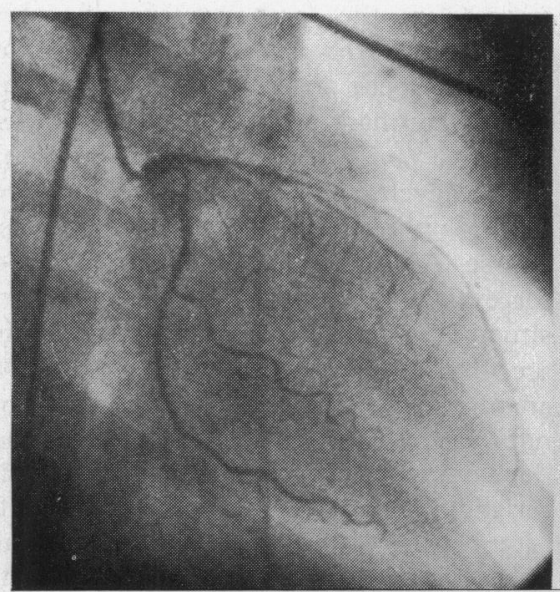

RAO

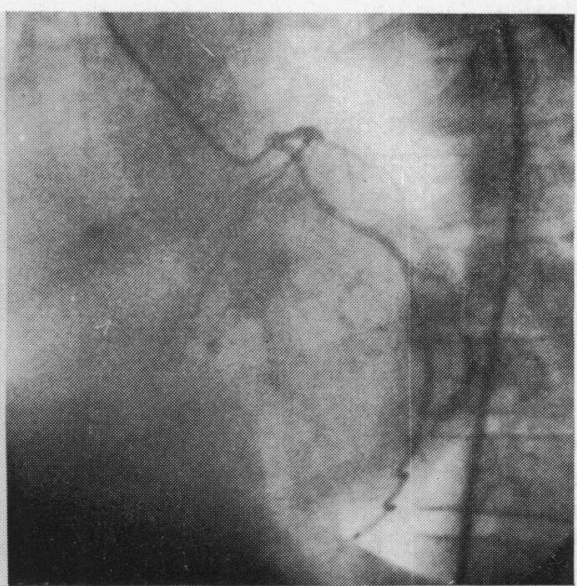

LAO

FIG. 3 Coronary arteriogram of a case with severe atherosclerotic changes in the branches of the left coronary artery. The main left coronary artery is short. Abbreviations as in Fig. 2. 
main left coronary artery but a longer artery does not exclude the occurrence of left bundle-branch block.

The following mechanism has been proposed as an explanation for the development of the left bundle-branch block in the presence of a short main left coronary artery (Lewis et al., 1970). The early septal branches of the left anterior descending artery are normally protected from shearing forces by the relative 'binding down' of the parent vessel. When the main left coronary artery is short, it is followed by a long initial unattached part of the left anterior descending artery which is subjected through systolic kinking to unusual stress. Under these circumstances, the blood flow to the left bundle, provided mainly by the first septal branch of the left anterior descending artery (James and Burch, 1958; Frink and James, 1973), is impaired.

The frequently observed localization of atherosclerotic changes in the initial part (or 'neck') of the left anterior descending artery has also been attributed to systolic motion. This part of the vessel, tethered proximally only by the main left coronary artery and distally by its first septal branch, is subjected to bending or kinking which are believed to enhance the development of atherosclerosis (Fulton, 1965; Glagov, 1972). It is conceivable that the longer the main left coronary artery the shorter the 'neck' of the left anterior descending artery and vice versa. Thus, a long main left coronary artery indirectly protects the 'neck' of the left anterior descending artery by reducing its loose pliable part and consequently its ability to bend during systole. This may be the best explanation for the frequent coexistence of left bundle-branch block and coronary atherosclerosis (Smith and Hayes, 1965; Mulcahy, Hickey, and Maurer, 1968; Lev et al., 1974). If these assumptions are correct a short main left coronary artery regardless of the development of conduction disturbances, would be expected to increase the chance of development of atherosclerosis at the origin of the left anterior descending artery. To our knowledge such a relation has not yet been reported, but the present findings support this hypothesis.

In addition to these views some other mechanisms may be involved in the development of atherosclerosis in the left coronary artery branches in the presence of a short main left coronary artery. According to Poiseuille's equation the drop in pressure and flow in a vessel is directly proportional to its length, while according to Young's formula (Moulopoulos, 1962) the modulus of elasticity and, therefore, its damping effect on pressure and flow changes are also directly proportional to the length of the vessel. Increased pressure, increased flow, and variables related to changes of pressure and flow have been considered as atherogenic hydraulic factors acting mainly on sites of bifurcation, branching, bending, and narrowing (Glagov, 1972; Fry, 1973; Texon, 1974). A long main left coronary artery may, therefore, be expected to protect the bifurcation of the vessel and the proximal part of its branches from the atherogenic effect of mechanical forces by reducing pressure and flow rate and by damping pulsatile pressure and flow. These hydraulic changes would be expected to affect not only the left arterior descending artery but also the circumflex artery.

It is known that in the presence of left ventricular hypertrophy, length and diameter of coronary arteries increase (Linzbach, 1960; Lewis and Gotsman, 1973), and it might therefore be assumed that the observed differences in the mean length of the main left coronary artery could be caused by the high proportion of cases (12 of 19) with left ventricular hypertrophy in the group of non-coronary patients. However, as seen in Fig. 1, there was no difference between cases with left ventricular hypertrophy and those without. A relation between the length of the main left coronary artery and the presence of left ventricular hypertrophy was not found by other investigators (Green, Bernstein, and Reppert, 1967). Furthermore, even after exclusion of patients with left ventricular hypertrophy, the difference in mean length between coronary and non-coronary patients remained significant. It seems that if left ventricular hypertrophy causes some elongation of the main left coronary artery, this effect must be minimal.

From a large variety of suggested aetiological factors in the pathogenesis of atherosclerosis, mechanical stresses and haemodynamic changes resulting from anatomical variations in the structure of coronary arteries have been considered as a sine qua non. Other known factors predisposing to the development of atherosclerosis were thought to be only secondary or merely modifying its course (Altschule, 1974). The presence of lipaemia, for example, would enhance atherosclerotic lesions situated at areas where local mechanical stresses are likely to be greatest (Glagov, 1972). Since anatomical structure and distribution of the coronary tree is possibly determined by heredity and development, as are other morphological characteristics, the length of the main left coronary artery trunk should be regarded as an inherited characteristic influencing the rate of development of coronary atherosclerosis.

\section{References}

Altschule, M. D. (1974). The etiology of atherosclerosis. 
Medical Clinics of North America, 58, 397.

Baroldi, G., and Scomazzoni, G. (1967). Coronary Circulation in the Normal and the Pathologic Heart, pp. 9, 35. Armed Forces Institute of Pathology, Washington D.C.

Beach, T. B., Gracey, J. G., Peter, R. H., and Grunenwald, P. W. (1969). Benign left bundle-branch block. Annals of Internal Medicine, 70, 269.

Fox, C., Davies, M. J., and Webb-Peploe, M. M. (1973). Length of left main coronary artery. British Heart fournal, 35, 796.

Frink, R. J., and James, T. N. (1973). Normal blood supply to the human His bundle and proximal bundle branches. Circulation, 47, 8.

Fry, D. L. (1973). Responses of the arterial wall to certain physical factors. In Atherogenesis: Initiating Factors. Ciba Foundation Symposium, p. 93. Ed. by R. Porter and J. Knight. Elsevier, Amsterdam.

Fulton, W. F. M. (1965). The Coronary Arteries, p. 64. Thomas, Springfield, Illinois.

Glagov, S. (1972). Hemodynamic risk factors: mechanical stress, mural architecture, medial nutrition and the vulnerability of arteries to atherosclerosis. In The Pathogenesis of Atherosclerosis, p. 164. Ed. by R. W. Wissler and J. C. Geer. Williams and Wilkins, Baltimore.

Green, G. E., Bernstein, S., and Reppert, E. H. (1967). The length of the left main coronary artery. Surgery, 62, 1021.

Haft, J. I., Herman, M. V., and Gorlin, R. (1969). Coronary arteriographic and left ventricular motion studies in left bundle branch block. American fournal of Cardiology, 23, 117.

Hamby, R. J., Tabrah, F., and Gupta, M. (1973). Intraventricular conduction disturbances and coronary artery disease. Clinical, hemodynamic and angiographic study. American fournal of Cardiology, 32, 758.

James, T. N., and Burch, G. E. (1958). Blood supply of the human interventricular septum. Circulation, 17, 391.

Kronzon, I., Deutsch, P., and Glassman, E. (1974). Length of the left main coronary artery. American fournal of Cardiology, 34, 787.

Lev, M., Unger, P. N., Rosen, K. M., and Bharati, S. (1974). The anatomic substrate of complete left bundle branch block. Circulation, 50, 479.

Lewis, B. S., and Gotsman, M. S. (1973). Relation between coronary artery size and left ventricular wall mass. British Heart fournal, 35, 1150.

Lewis, C. M., Dagenais, G. R., Friesinger, G. C., and Ross, R. S. (1970). Coronary arteriographic appearances in patients with left bundle-branch block. Circulation, 41, 299.

Linzbach, A. J. (1960). Heart failure from the point of quantitative anatomy. American fournal of Cardiology, 5, 370.

Moulopoulos, S. D. (1962). Cardiomechanics, p. 33. Thomas, Springfield, Illinois.

Mulcahy, R., Hickey, N., and Maurer, B. (1968). Aetiology of bundle-branch block. British Heart fournal, 30, 34 .

Smith, S., and Hayes, W. L. (1965). The prognosis of complete left bundle-branch block. American Heart fournal, 70, 157.

Texon, M. (1974). Atherosclerosis. Its hemodynamic basis and implications. Medical Clinics of North America, 58, 257.

Requests for reprints to Dr. N. Gazetopoulos, Cardiology Division, Aretaeion Hospital, 76 Vas. Sophias Avenue, Athens 611, Greece. 